\title{
Lisdexamfetamine Dimesylate for Adults with Moderate to Severe Binge Eating Disorder: Results of Two Pivotal Phase 3 Randomized Controlled Trials
}

\author{
Susan L McElroy ${ }^{*, 1,2}$, James Hudson ${ }^{3,4}$, M Celeste Ferreira-Cornwell ${ }^{5}$, Jana Radewonuk ${ }^{6}$, Timothy Whitaker ${ }^{5}$ \\ and Maria Gasior ${ }^{5}$ \\ 'Lindner Center of HOPE, Mason, OH, USA; ${ }^{2}$ Department of Psychiatry and Behavioral Neuroscience, University of Cincinnati College of Medicine, \\ Cincinnati, OH, USA; ${ }^{3}$ Biological Psychiatry Laboratory, McLean Hospital, Belmont, MA, USA; ${ }^{4}$ Department of Psychiatry, Harvard Medical School, \\ Belmont, MA, USA; ${ }^{5}$ Global Clinical Development, Shire, Wayne, PA, USA; ${ }^{6}$ Clinical Development Operations \& Biometrics, Shire, Wayne, PA, USA
}

\begin{abstract}
The efficacy and safety of lisdexamfetamine dimesylate (LDX) vs placebo in binge eating disorder (BED) was evaluated in two multicenter, double-blind, placebo-controlled trials. Adults (study I, $n=383$; study 2, $n=390$ ) meeting DSM-IV-TR BED criteria were randomized $(|:|$ ) to placebo or LDX (50 or 70 mg/day) dose titration; optimized doses were maintained to the end of double-blind treatment (week I2/ early termination). Change from baseline in binge eating days/week at weeks 1 I- 12 (primary efficacy endpoint) was assessed with mixedeffects models for repeated measures. Secondary endpoints related to binge eating and medical parameters, safety, and treatment compliance were also assessed. Least squares mean $(95 \% \mathrm{Cl})$ treatment differences for change from baseline binge eating days/week at weeks II-12 significantly favored LDX (study I: $-1.35[-1.70,-1.0$ I]; study $2:-1.66[-2.04,-1.28]$; both $P<0.00$ I). In both studies, treatment-emergent adverse events (TEAEs) reported by $\geqslant 10 \%$ of LDX participants were dry mouth, insomnia, and headache. Serious TEAEs occurred in two ( $1.1 \%$ ) placebo participants in each study and in three (1.6\%) and one (0.6\%) LDX participants in study I and study 2, respectively. Across studies, mean increases from baseline at week 12/early termination with LDX for pulse and systolic and diastolic blood pressure ranged from 4.4 I-6.3 I b.p.m. and $0.2-1.45$ and $1.06-1.83 \mathrm{~mm} \mathrm{Hg}$, respectively. LDX (50 and 70 mg/day) was superior to placebo in decreasing binge eating days/week from baseline and improving binge eating-related key secondary endpoints. Safety results appear consistent with the known safety profile of LDX.

Neuropsychopharmacology (2016) 4I, I25 I-1260; doi:I0.I038/npp.20I5.275; published online 28 October 20I5
\end{abstract}

\section{INTRODUCTION}

The Diagnostic and Statistical Manual of Mental Disorders, Fifth Edition (DSM-5) recognizes binge eating disorder (BED) as a distinct disorder (American Psychiatric Association, 2013). In BED, binge eating episodes occur $\geqslant 1$ time per week for $\geqslant 3$ months; in the DSM-IV-TR provisional BED criteria, binge eating occurs on average $\geqslant 2$ days per week for $\geqslant 6$ months (American Psychiatric Association, 2000). During these episodes, larger than usual amounts of food are consumed during a discreet time period and there is subjective lack of control and marked distress over eating. Unlike in bulimia nervosa and anorexia nervosa, there are no recurrent and inappropriate compensatory behaviors in BED, such as excessive exercise or purging.

The estimated lifetime prevalence of BED is $~ 2-3 \%$ (Hudson et al, 2007; Kessler et al, 2013). Individuals with BED have been reported to be at increased risk for obesity

\footnotetext{
*Correspondence: Dr SL McElroy, Lindner Center of HOPE, 4075 Old Western Row Rd, Mason, OH 45040, USA, Tel: + I 5 I 3 536-0700, Fax: + I 513 536-0709, E-mail: susan.mcelroy@lindnercenter.org

Received I April 2015; revised 2 July 2015; accepted 7 July 2015; accepted article preview online 9 September 2015
}

and psychiatric comorbidities (Hudson et al, 2007; Kessler et al, 2013); to be at increased risk for developing components of metabolic syndrome, including dyslipidemia and type 2 diabetes, even after controlling for body mass index (BMI) (Hudson et al, 2010); to have reduced healthrelated quality of life (Masheb and Grilo, 2004); and to have increased healthcare utilization (Striegel-Moore et al, 2004). Evidence suggests that binge eating pathology in BED may respond to psychotherapy and pharmacotherapy (Reas and Grilo, 2014; Vocks et al, 2010 ). Given the prevalence and consequences of BED, more treatment options are needed.

Lisdexamfetamine dimesylate (LDX), a d-amphetamine prodrug (Pennick, 2010) indicated for the treatment of attention-deficit/hyperactivity disorder, is also now approved in the United States for the treatment of adults with moderate to severe BED (Vyvanse [lisdexamfetamine dimesylate], 2015). In a phase 2, fixed-dose, randomized, multicenter, double-blind, parallel-group, placebo-controlled, dose-finding study, LDX (50 and $70 \mathrm{mg} /$ day but not $30 \mathrm{mg} /$ day) demonstrated efficacy $v s$ placebo in decreasing binge eating days in individuals with BED (McElroy et al, 2015). Here, the efficacy, safety, and tolerability findings of two phase 3, randomized, multicenter, double-blind, parallel-group, placebo-controlled trials in adults with 
protocol-defined BED of at least moderate severity are reported.

\section{MATERIALS AND METHODS}

\section{Trial Design}

Two randomized (1:1), placebo-controlled, parallel-group, multicenter studies (Study 343, NCT01718483 [referred to as study 1 hereafter]; Study 344, NCT01718509 [referred to as study 2 hereafter]) were conducted using the same design and methods. Study 1 included 50 unique sites (United States, $n=44$; Sweden, $n=3$; Spain, $n=1$; Germany, $n=2)$ between 26 November 2012 and 25 September 2013. Study 2 included 43 unique sites (United States, $n=41$; Germany, $n=2$ ) between 26 November 2012 and 20 September 2013. In studies 1 and 2, the mean number of randomized participants per site was 7.7 (median, 7; range, 1-24) and 9.1 (median, 8; range, 1-25), respectively. For each site, there was only one principal investigator but multiple clinicians may have been involved at any individual site. Qualified clinicians were trained and approved to ensure rigor, validity, and standardization. Standardized training was provided by an expert in BED and included training on the $D S M-I V-T R$ criteria for BED, the core symptoms of BED, the definition of a binge eating episode, and on the completion, content, and interpretation of the daily binge diary.

Subsequent to study completion and before unblinding, two sites were excluded from study 2 . One site was excluded for Good Clinical Practice non-compliance; efficacy data were excluded and safety data were included in primary analyses. One additional site was excluded due to an initiated external investigation for reasons unrelated to the respective study; safety and efficacy data from this site were excluded from primary analyses.

Each study included 2- to 4-week screening, 12-week double-blind treatment (dose optimization, 4 weeks; dose maintenance, 8 weeks), and 1-week follow-up phases (Figure 1a). Dose-optimization designs, with target doses established in the phase 2 study (50 and $70 \mathrm{mg}$ LDX) (McElroy et al, 2015), were used. Study protocols were approved by ethics committees; both studies were conducted in accordance with International Conference on Harmonisation Good Clinical Practice and the principles of the Declaration of Helsinki. Participants were required to provide written, informed consent before entering the studies.

\section{Participants}

Participants were recruited from investigators' databases and via local/central advertisement. Eligible participants were men or nonpregnant women (18-55 years). These studies were conducted before the release of the DSM-5, which states that the minimum level of BED severity should be based on the frequency of episodes of binge eating and that the severity level may be increased to reflect other symptoms and the degree of functional disability (American Psychiatric Association, 2013). These studies implemented dual criteria for protocol-defined moderate to severe BED that were based on DSM-IV-TR criteria and agreed upon by the authors.
These dual criteria designated moderate to severe BED as the presence of a binge eating frequency of $\geqslant 3$ binge eating days/week for 2 consecutive weeks before baseline and a Clinical Global Impressions-Severity score (Guy, 1976) at screening and baseline of $\geqslant 4$. These criteria were considered clinically relevant because, in addition to accounting for binge eating frequency, they incorporate a global assessment of binge-related symptoms, distress, and impairment. BED diagnosis was confirmed by the eating disorders module of the Structured Clinical Interview for the DSM-IV-TR Axis I Disorders (SCID-I) (First et al, 2007) and the Eating Disorder Examination Questionnaire (Fairburn and Beglin, 1994). At screening and baseline, eligible participants had $\mathrm{BMI} \geqslant 18$ and $\leqslant 45$.

Key exclusion criteria included: current anorexia nervosa (AN) or bulimia nervosa (BN), as defined by the SCID-I eating disorders module; comorbid current psychiatric disorders either controlled with prohibited medications or uncontrolled and associated with significant symptoms or any condition/symptom that may confound clinical assessment; psychotherapy or weight loss support (including peer support) for BED $\leqslant 3$ months before screening (psychotherapy for conditions other than BED was not recorded); use of psychostimulants for fasting or dieting for BED $\leqslant 6$ months before screening; Montgomery-Åsberg Depression Rating Scale total score $\geqslant 18$ at screening; being considered a suicide risk by the investigator, having previously made a suicide attempt, or currently demonstrating active suicidal ideation; lifetime histories of psychosis, mania, hypomania, dementia, or attention-deficit/hyperactivity disorder; histories of symptomatic cardiovascular disease, structural cardiac or heart rhythm abnormalities, cardiomyopathy, or coronary artery disease; moderate or severe hypertension, resting average sitting systolic blood pressure $>139 \mathrm{~mm} \mathrm{Hg}$, or average diastolic blood pressure $>89 \mathrm{~mm} \mathrm{Hg}$ at screening or baseline (mild, controlled hypertension was not exclusionary); a clinically significant electrocardiogram (ECG) at screening or baseline; lifetime amphetamine or stimulant abuse/dependence histories; recent history of substance abuse/dependence (except nicotine); and having known/ suspected intolerance or hypersensitivity to LDX or related compounds.

\section{Intervention}

After a 2- to 4-week screening period, eligible participants were randomized 1:1 to 12 weeks of dose-optimized LDX or matching placebo (Figure 1a). The randomization schedule was assigned by an interactive web response system. For blinding, both treatments were identical in appearance; the blind was not to be broken during the study.

During week 1, LDX was given at a daily dosage of $30 \mathrm{mg}$ for initial titration only. During week 2, the daily LDX dosage was titrated to $50 \mathrm{mg}$. During weeks $3-4$, increases to $70 \mathrm{mg}$ LDX were made based on tolerability and clinical need. A single downward titration to $50 \mathrm{mg}$ was allowed at week 3 if the $70-\mathrm{mg}$ dose was not tolerated. During weeks $4-12$, the optimized LDX dosage (50 or $70 \mathrm{mg}$ ) was maintained. If a dose reduction occurred during optimization phase, no further changes were allowed during maintenance; participants requiring such a reduction were 
a

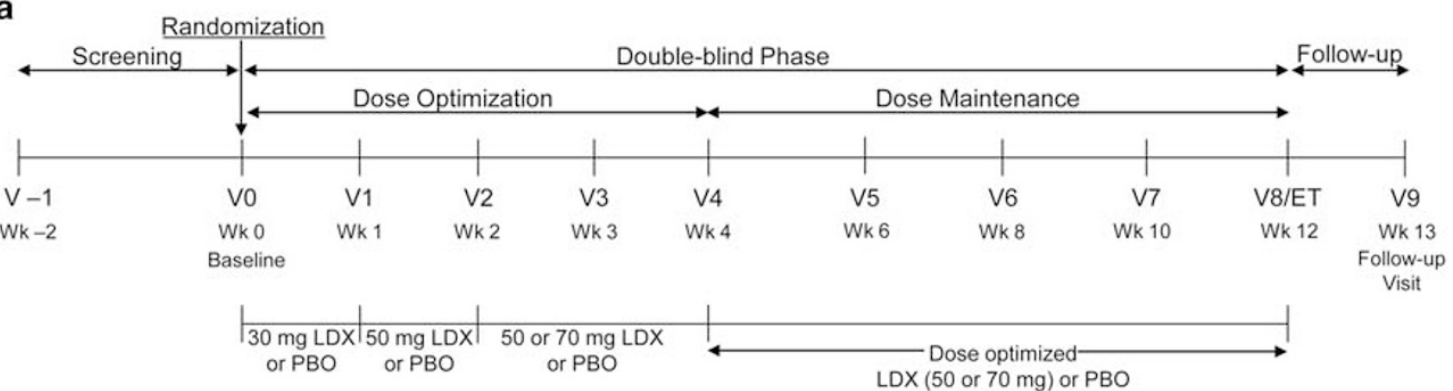

$\mathrm{ET}=$ early termination; $\mathrm{PBO}=$ placebo; $\mathrm{V}=$ visit; $\mathrm{Wk}=$ week

b

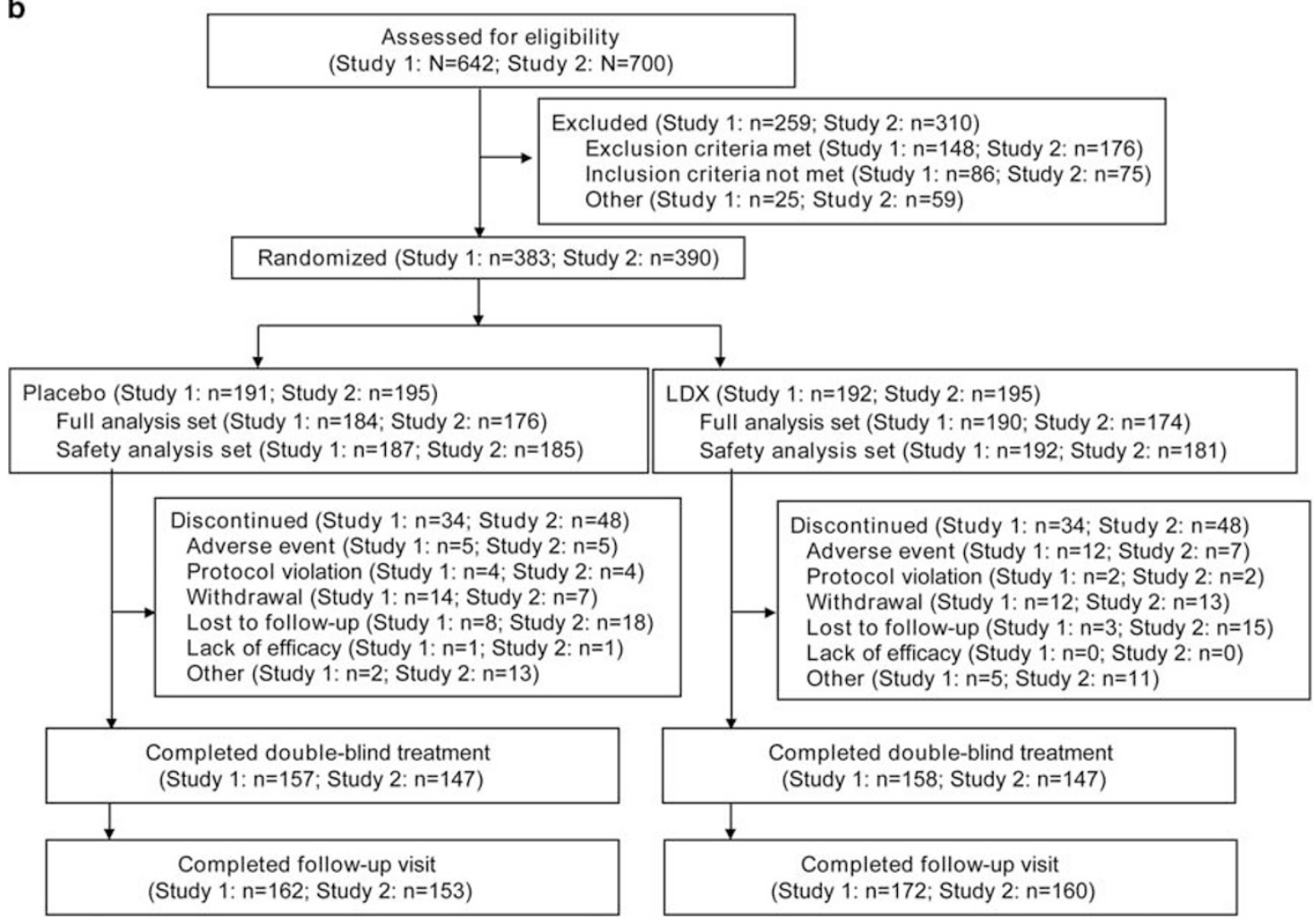

Figure I Study timeline and titration schedule (a) and participant disposition (b).

discontinued from the study. Throughout the study, participants were instructed to consume one capsule daily at $\sim 0700$ hours $( \pm 2 \mathrm{~h})$. A follow-up visit occurred 1 week after week 12 /early termination (ET).

\section{Efficacy Assessments}

The primary efficacy endpoint was change from baseline in binge eating days/week at weeks 11-12 (visit 8) based on participants' daily binge eating diaries, as reviewed and confirmed by investigators, as previously reported in a phase 2 study (McElroy et al, 2015). To allow for visit windows of \pm 2 days and for missing diary days, binge eating days/week was the number of confirmed binge eating days between visits multiplied by 7 and divided by the number of nonmissing diary days during the period. Binge eating days were recorded in daily participants' diaries and assessed by clinicians at all study visits except screening.
Prespecified key secondary endpoints assessed global BED improvement (CGI-Improvement; CGI-I) at week 12/ET, proportion of participants with 4 -week binge eating cessation at week 12/ET (no binge eating episodes for 28 consecutive days before the last visit), Yale-Brown Obsessive Compulsive Scale Modified for Binge Eating (Y-BOCS-BE) total score changes from baseline at week 12), and medical aspects (percentage weight change from baseline at week 12 and fasting triglyceride changes from baseline to week 12/ET) of BED. CGI-I scores were assessed at weeks 1-4, 6, 8, 10, and $12 / E T$. Body weight was assessed on a calibrated scale (without shoes) at all study visits. The Y-BOCS-BE, a modified version of Y-BOCS (Goodman et al, 1989) that has previously been used in BED pharmacotherapy studies (McElroy et al, 2003, 2007a, b), was assessed at baseline and weeks 4, 8, and 12/ET. The Y-BOCS-BE assesses obsessiveness of binge eating thoughts and compulsiveness of binge eating behaviors using a 10-item clinician-rated 
scale ( 0 [no symptoms] to 4 [extreme symptoms]); total scores range from $0-40$. Fasting triglycerides were assessed at screening and week 12/ET.

\section{Safety and Tolerability Assessments}

Safety and tolerability assessments included adverse events (AEs), vital signs, weight, ECGs, clinical laboratories, and scores on the Columbia-Suicide Severity Rating Scale (C-SSRS) and Amphetamine Cessation Symptom Assessment (ACSA). The C-SSRS is a clinician-administered prospective assessment of suicidal ideation and behavior (Posner et al, 2011). The ACSA, a self-completed questionnaire, contains 16 items rated on 5-point scales (0 [not at all] to 4 [extremely]); total score ranges from 0-64 (McGregor et al, 2008). Adverse events, vital signs, weight, and C-SSRS scores were assessed at all study visits. Scores on the ACSA were assessed at baseline, week 12/ET, and daily through the follow-up period. Clinical laboratory evaluations were assessed at screening and week 12/ET; ECGs were assessed at screening, baseline, week 4 , and week 12/ET. Clinical adherence (participants taking $80-100 \%$ of study medication) was determined by the investigator at every visit for study management purposes; calculated adherence (participants taking $80-120 \%$ of study medication: total capsules taken $\times 100 /$ total days of dosing) was determined as a statistical compliance assessment.

\section{Statistical Analysis}

Statistical assessments of the primary and key secondary efficacy endpoints were conducted in the full analysis set (all randomized participants taking $\geqslant 1$ study drug dose and having $\geqslant 1$ postbaseline primary efficacy assessment); statistical significance was set at a 2 -sided $P<0.05$. Hierarchical testing procedures were used, with statistical assessments made in the following order based on clinical importance and likelihood of effect based on phase 2 results: changes in binge eating days/week, CGI-I, 4-week binge eating cessation, percentage body weight changes, Y-BOCS$\mathrm{BE}$ total score changes, and triglyceride changes. A later test was only significant if all earlier tests were significant.

Sample size was estimated for the primary efficacy endpoint using nQuery 6.0 (Statistical Solutions; Boston, MA). Assuming an effect size of 0.4 (LDX $v s$ placebo), it was estimated that 133 participants completing each treatment would provide $90 \%$ power.

Change in binge eating days/week was assessed using a mixed-effects model for repeated measures (MMRM), with treatment, visit, and the treatment $\times$ visit interaction as factors and baseline binge eating days/week as a covariate; the baseline binge eating days/week $\times$ visit interaction was also included in the model. MMRM accounts for the repeated measures structure of the data by factoring in correlations within each participant across multiple visits (Siddiqui, 2011) and is an analytic method that is widely accepted for continuous endpoints in clinical trials. The primary efficacy analysis was based on the assumption of a missing-at-random mechanism, namely, missingness was not related to the data not observed (Little et al, 2012). Two preplanned sensitivity analyses were conducted using a different missing-not-at-random mechanism, which assumes that missingness was related to the data observed (Little et al, 2012). The first preplanned sensitivity analysis model used multiple imputations based on the distribution of placebo responses over time; the second model used multiple imputations with penalties applied to participants who discontinued.

Dichotomized CGI-I scores of improved (very much improved or much improved) vs not improved (minimally improved to very much worse) and the proportion of participants attaining 4-week binge eating cessation at week 12/ET were assessed with $\chi^{2}$ tests and CIs for binomial proportions. Y-BOCS-BE total score changes and percentage weight changes from baseline to week 12 were assessed using MMRM, as described above. Change from baseline to week $12 / \mathrm{ET}$ in triglycerides was assessed using analysis of covariance, with treatment as a factor and baseline as a covariate. Safety and tolerability assessments were conducted in the safety analysis set (all randomized participants taking $\geqslant 1$ study drug dose and having $\geqslant 1$ postbaseline safety assessment) and are presented descriptively.

Post hoc sensitivity analyses were also conducted that included data from the two aforementioned excluded sites using the same statistical methods described above.

\section{RESULTS}

\section{Disposition and Demographics}

Most participants from each treatment group completed each study (Figure 1b). Demographic and clinical characteristics are summarized in Table 1 . In both studies, most participants were white, female, and obese $\left(B M I \geqslant 30.0 \mathrm{~kg} / \mathrm{m}^{2}\right)$. The baseline mean \pm SD number of binge eating days/week was $4.69 \pm 1.237$ in study 1 and $4.75 \pm 1.359$ in study 2 .

The overall proportion of individuals with comorbid psychiatric disorders was low, with the most common psychiatric disorder in each study being past major depressive disorder (Supplementary Table 1). Few participants had received psychotherapy for BED in the past (study 1: placebo, 2.1\% [4/187]; LDX, $1.6 \%$ [3/192]; study 2: placebo, $1.1 \%$ [2/185]; LDX, $0 \%$ ) or were currently receiving psychotherapy for BED (study 1: placebo, 0.5\% [1/187]; LDX, $0 \%$; study 2: placebo, $0.5 \%$ [1/185]; LDX, $0.6 \%$ [1/181]).

No participant in either study met criteria for a current diagnosis of $\mathrm{BN}$ or $\mathrm{AN}$ or for diagnoses of $\mathrm{BN}$ or AN within the last month. In study 1 , two participants reported lifetime histories of AN (at least 58 months had elapsed since the last symptoms) and six participants reported lifetime histories of BN (at least 18 months had elapsed since the last symptoms). In study 2, one participant reported a lifetime history of AN (at least 72 months had elapsed since the last symptoms) and two participants reported lifetime histories of BN (at least 36 months had elapsed since the last symptoms).

\section{Drug Exposure and Adherence}

The mean \pm SD daily LDX dosage during the entire doubleblind treatment phase was $56.9 \pm 9.72 \mathrm{mg}$ (study 1) and $57.6 \pm 9.24 \mathrm{mg}$ (study 2). Most LDX participants received $70 \mathrm{mg}$ as the optimized dosage (study 1, 117/192 [60.9\%]; 
Table I Demographics and Baseline Clinical Characteristics, Safety Analysis Set

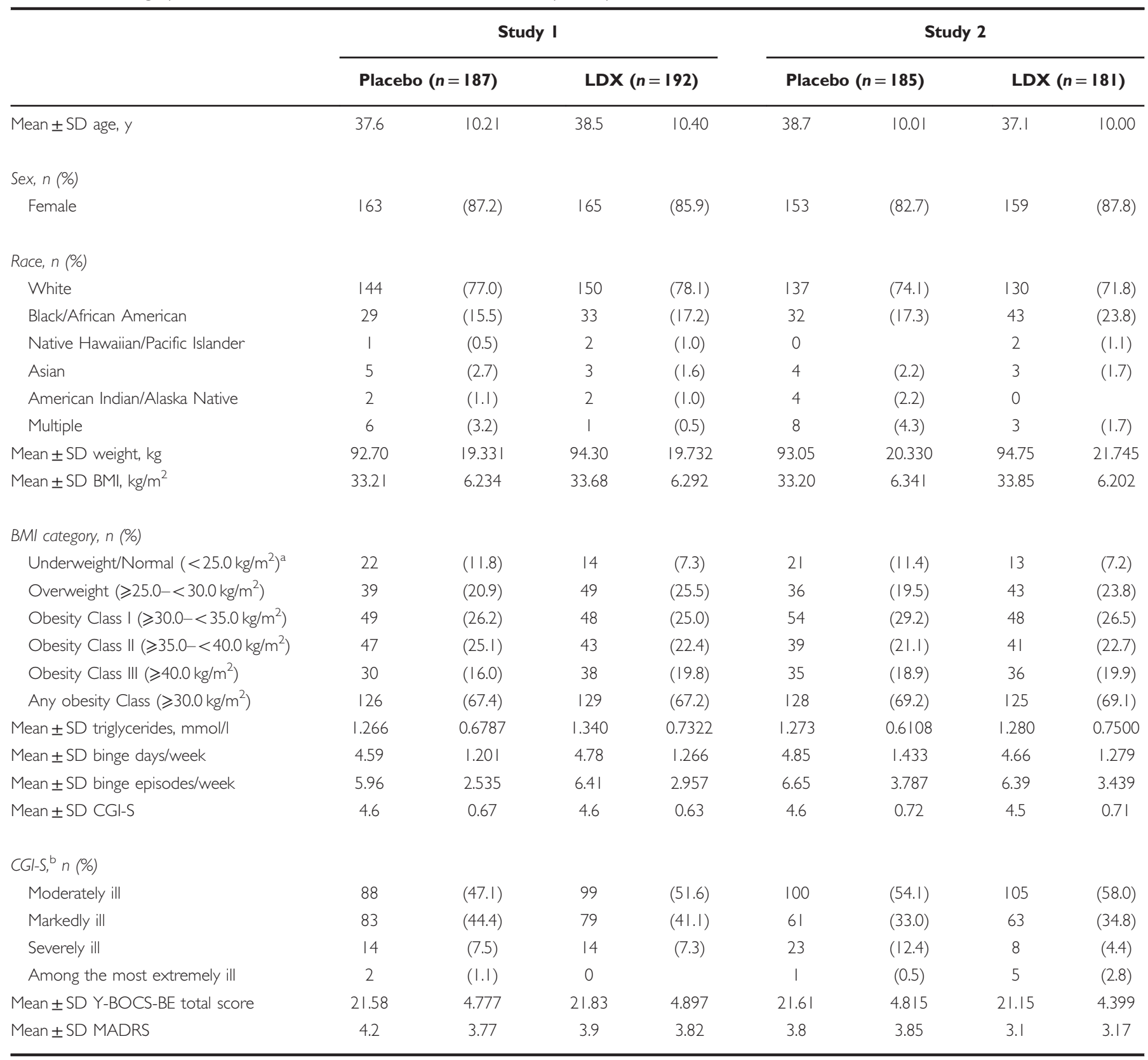

Abbreviations: BMI, body mass index; CGI-S, Clinical Global Impressions-Severity; LDX, lisdexamfetamine dimesylate; MADRS, Montgomery-Åsberg Depression Rating Scale; Y-BOCS-BE, Yale-Brown Obsessive Compulsive Scale Modified for Binge Eating.

aparticipants with $\mathrm{BMI}<18 \mathrm{~kg} / \mathrm{m}^{2}$ were not enrolled.

${ }^{b}$ Based on inclusion criteria, a CGI-S score $\geqslant 4$ (at least moderately ill) was required for study eligibility.

study 2, 113/181 [62.4\%]); $50 \mathrm{mg}$ was the optimized dosage in $29.7 \%(57 / 192)$ and $28.7 \%(52 / 181)$ of LDX participants in study 1 and study 2, respectively. Mean (SD) days of exposure (placebo vs LDX) were $76.6 \pm 20.72$ and $75.7 \pm 20.81$ in study 1 and $73.1 \pm 22.99$ and $75.8 \pm 20.14$ in study 2 .

Most participants were regarded as clinically adherent; calculated adherence was high (study 1: placebo $=187 / 187$ [100\%], LDX $=188 / 192$ [97.9\%]; study 2: placebo $=183 / 185$ [98.9\%], LDX $=180 / 181[99.4 \%])$. No participant in either study took $>120 \%$ of the medication.

\section{Efficacy}

Primary endpoint. The least squares mean (SEM) changes from baseline in binge eating days/week at weeks $11-12$ were $-2.51 \quad(0.125)$ with placebo and -3.87 (0.124) with LDX in study 1 and $-2.26(0.137)$ with placebo and $-3.92(0.135)$ with LDX in study 2 (Figure $2 \mathrm{a}$ and $\mathrm{b}$; see Table 1 for baseline values); least squares mean (95\% CI) treatment differences for change from baseline at weeks $11-12$ favored LDX for both studies (study 1: -1.35 $[-1.70,-1.01], P<0.001$; effect size $[95 \% \mathrm{CI}], 0.83[0.60$, 


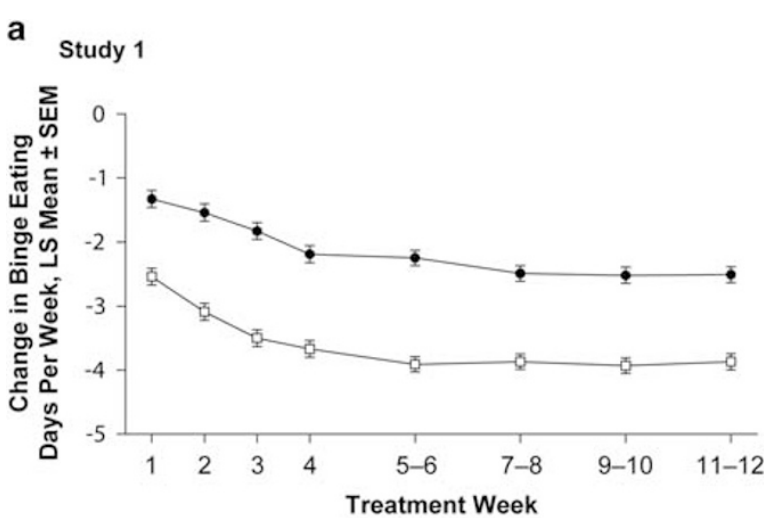

b

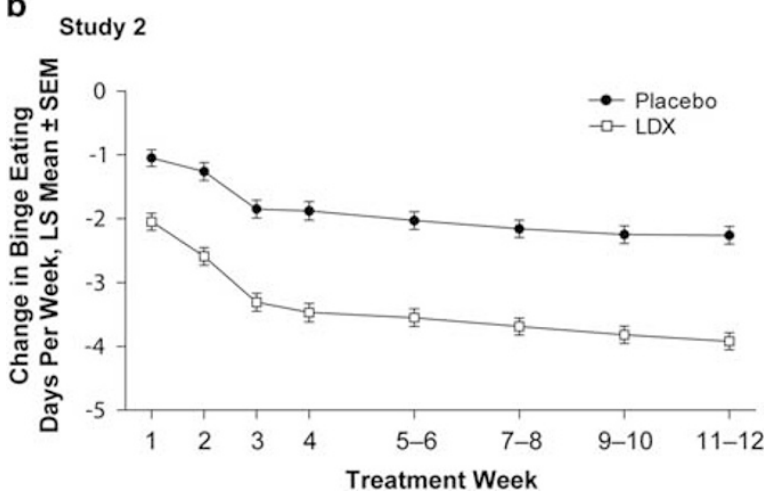

Figure 2 Change from baseline in binge eating days per week in Study I (a) and Study 2 (b), full analysis set. LDX, lisdexamfetamine dimesylate.

1.05]; study 2: -1.66 [-2.04, -1.28$], P<0.001$; effect size [ $95 \%$ CI], $0.97[0.72,1.21])$.

The pattern of missing values and the results of the preplanned sensitivity analyses based on missing-not-atrandom assumptions for the change from baseline in the number of binge days per week are summarized in Supplementary Tables 2 and 3, respectively. The results of the preplanned sensitivity analysis were consistent with the primary analysis results, indicating the data are robust to underlying missingness assumptions.

Subgroup analyses of the primary efficacy endpoint by age ( $<40 v s \geqslant 40$ years), sex, and race (white $v s$ nonwhite) are depicted in Supplementary Figure 1. In all subgroups, least squares mean decreases from baseline in the number of binge eating days/week were noted for both treatment groups and were numerically greater for LDX vs placebo. For the majority of subgroups, the 95\% CIs (except for the male and non-white subgroups in study 1) fell to the left of zero, indicating greater mean improvement for LDX relative to placebo. However, because randomization was not stratified based on these subgroups, the number of participants within each subgroup was not consistently balanced and definitive conclusions cannot be drawn.

Key secondary endpoints. Statistically significant treatment effects favoring LDX were seen for CGI-I, 4-week cessation, body weight, and Y-BOCS-BE in both studies (Table 2).
Differences in the reduction in triglyceride levels for LDX $v s$ placebo were also statistically significant in both studies (Table 2), with mean values being within the normal range at baseline and week 12/ET.

\section{Safety and Tolerability}

In each study, $>50 \%$ of the participants in each treatment group reported TEAEs (Table 3); more TEAEs were related to study drug with LDX than with placebo. Most TEAEs in each study were mild or moderate in severity. In each study, TEAEs reported by $>10 \%$ of LDX-treated participants were dry mouth, headache, and insomnia; no TEAE was reported in $>10 \%$ of placebo-treated participants (Table 3 ).

Serious TEAEs were infrequent and reported in equal proportions with LDX and placebo in each study (Table 3) and generally reflected intercurrent illness and accidental or potential BED-associated comorbidities. LDX-associated SAEs were considered unrelated to treatment by investigators, except for two syncope cases which resulted in participant discontinuation. One case did not require medical intervention; the other was confounded by multiple concomitant medications and a medical history of narcolepsy and hypertension. A single instance of syncope as a treatment-emergent SAE also occurred with placebo and resulted in participant discontinuation. TEAEs leading to discontinuation occurred infrequently in both groups in both studies (Table 3); there were no deaths during either study.

Across studies, mean increases from baseline at week 12/ET with LDX ranged from 4.41-6.31 b.p.m. for pulse rate, $0.2-1.45 \mathrm{~mm} \mathrm{Hg}$ for systolic blood pressure, and 1.06-1.83 mm Hg for diastolic blood pressure (Table 3). All baseline ECG measures were similar between treatments in each study, with ECG-assessed heart rate changes being similar to pulse rate changes (Table 3).

Mean \pm SD ACSA total aggregate scores were low at baseline in both groups (study 1: placebo $=9.6 \pm 8.63$, $\mathrm{LDX}=10.3 \pm 10.28$; study 2 : placebo $=7.6 \pm 8.87, \mathrm{LDX}=7.3$ \pm 8.46 ); scores were below baseline scores during the post-cessation period (day of last dose: placebo $=7.3 \pm 7.74$ and $\operatorname{LDX}=5.7 \pm 7.37$ in study 1 and placebo $=7.0 \pm 7.69$, LDX $=4.6 \pm 5.83$ in study 2); 7 days post treatment: placebo $=5.5 \pm 7.41$ and $\mathrm{LDX}=5.7 \pm 7.42$ in study 1 and placebo $=4.1 \pm 6.02$ and $\operatorname{LDX}=5.1 \pm 7.32$ in study 2 ), with no indication of withdrawal symptoms as measured by ACSA.

On the C-SSRS, there were no positive affirmations including preparatory acts, actual, interrupted, or aborted suicide attempts with either treatment during either study. There were positive affirmations of active suicidal ideation in study 1 only with placebo ("any active suicidal ideation": $n=1$ each at weeks 1 and 12). There were no positive affirmations of active suicidal ideation with either treatment in study 2 .

\section{Post Hoc Sensitivity Analyses}

The overall efficacy, safety, and tolerability findings of study 2 did not change based on the inclusion of data from the 2 aforementioned excluded study sites (data not shown). 
Table 2 Summary of Key Secondary Endpoints, Full Analysis Set

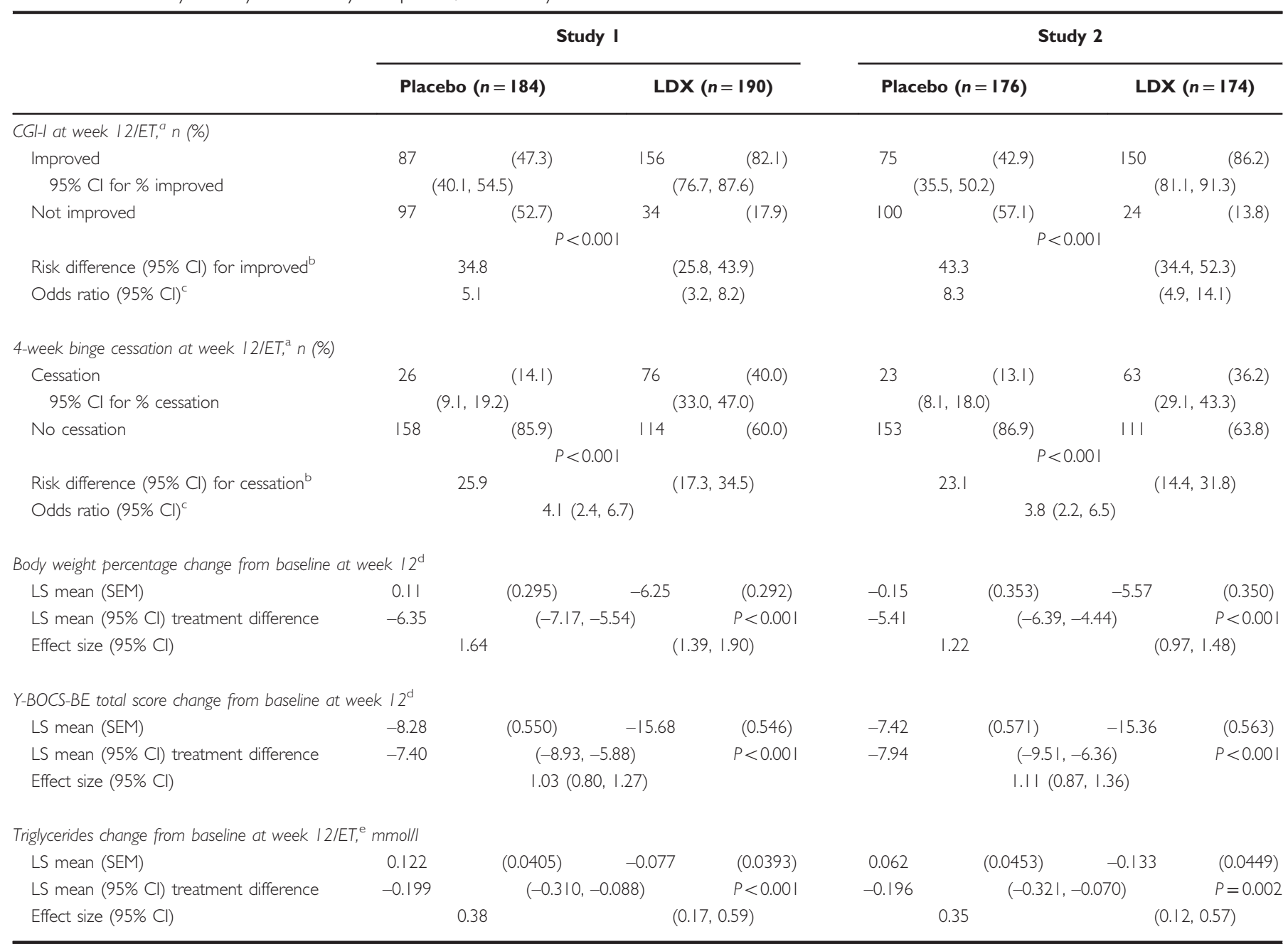

Abbreviations: CGI-I, Clinical Global Impressions-Improvement; ET, early termination; LDX, lisdexamfetamine dimesylate; LS, least squares; Y-BOCS-BE, Yale-Brown Obsessive Compulsive Scale Modified for Binge Eating.

${ }^{\text {a }}$-values based on $\chi^{2}$ tests; $95 \%$ Cls based on binomial proportions.

bifference calculated as LDX-placebo.

'Ratio calculated as LDX/placebo.

${ }^{\mathrm{d}} P$-values based on mixed-effects model for repeated measures, with treatment, visit, and the treatment $\times$ visit interaction as factors and baseline value as a covariate and the interaction of baseline $\times$ visit included in the model; effect size based on the estimated SD from the unstructured covariance matrix.

${ }^{\mathrm{e}} \mathrm{P}$-values based on analysis of covariance, with treatment as a factor and baseline value as a covariate; effect size based on the estimated SD from the root mean square error

\section{DISCUSSION}

LDX produced statistically significant and clinically meaningful reductions in binge eating days/week (primary efficacy endpoint) relative to placebo in adults with moderate to severe BED. This same effect was observed in both of the two identically designed studies. These results are consistent with previous findings from a phase 2 trial of LDX for the treatment of BED, in which 50 and $70 \mathrm{mg}$ LDX (but not $30 \mathrm{mg}$ ) produced significantly greater decreases in binge eating days/week than placebo (McElroy et al, 2015). In the current studies, LDX was also associated with statistically significant and clinically meaningful greater response on outcomes of global improvement in BED pathology, 4-week cessation of binge eating at endpoint, and BED-related obsessive and compulsive psychopathology.
In addition, the percent weight reduction from baseline with LDX was statistically significantly greater than with placebo. Reductions from baseline in fasting triglycerides were statistically greater with LDX compared with placebo, but the clinical significance of these changes is unclear because mean baseline and week12/ET values were in the normal range.

Although LDX is currently the only medication approved for the treatment of adults with mild to moderate BED by the US Food and Drug Administration (Vyvanse [lisdexamfetamine dimesylate], 2015), several other types of medications have been investigated for the treatment of BED. Placebo-controlled studies of antidepressants have usually, but not always, found a statistically significant reduction in the frequency of binge eating and related measures of 
Table 3 TEAEs and Vital Sign Changes From Baseline at Week 12/ET, Safety Analysis Set

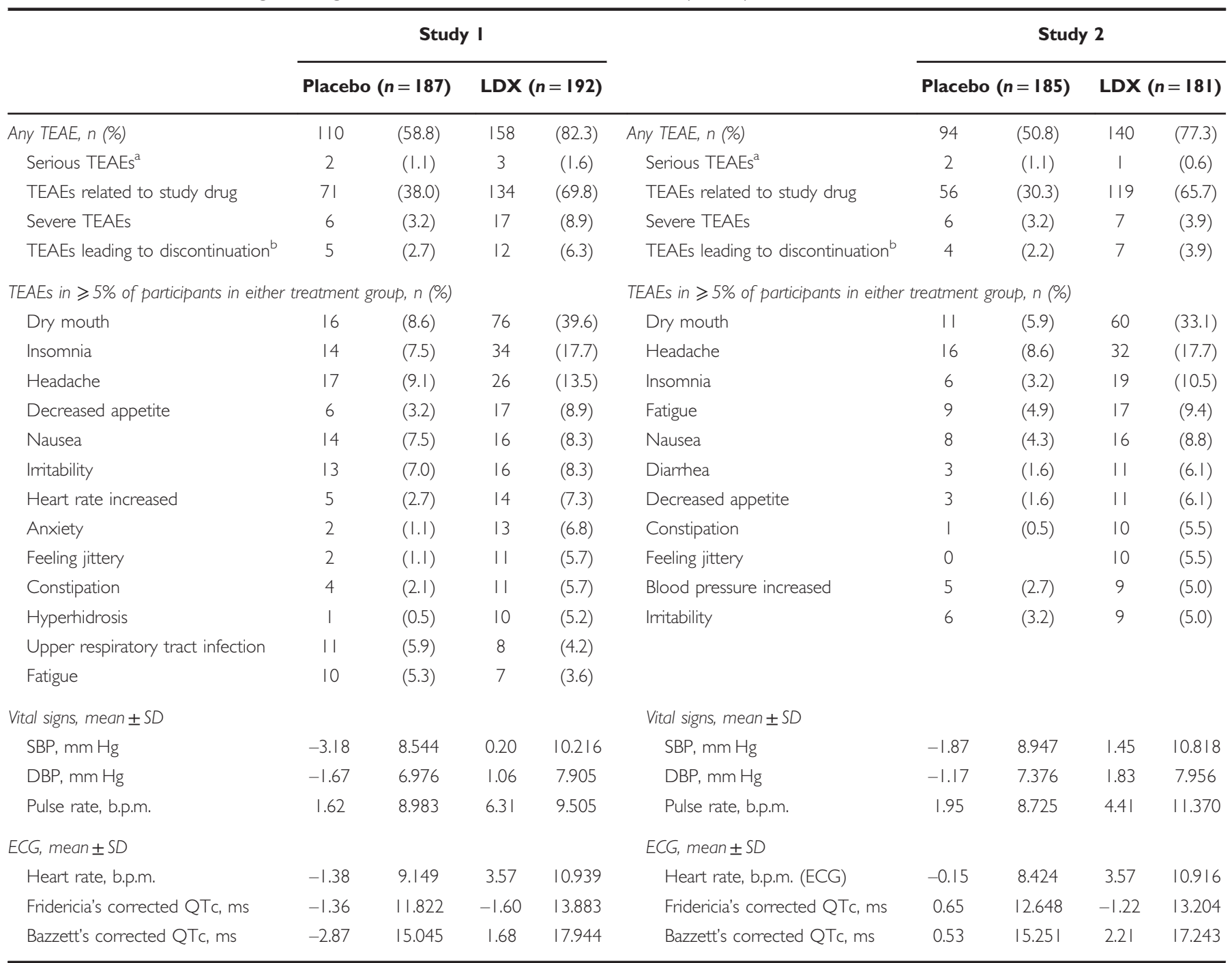

Abbreviations: b.p.m., beats per minute; DBP, diastolic blood pressure; ECG, electrocardiogram; ET, early termination; LDX, lisdexamfetamine dimesylate; SBP, systolic blood pressure; TEAE, treatment-emergent adverse event.

${ }^{a}$ Study I: anaphylactic reaction and conversion disorder (each 0.5\%) with placebo, syncope (I.0\%) and cholecystitis (0.5\%) with LDX; study 2: fibula fracture, syncope, agitation, and anxiety (each $0.5 \%$ ) with placebo, lumbar vertebral fracture $(0.6 \%)$ with LDX.

bStudy I: chest discomfort, palpitations, conversion disorder, anaphylactic reaction, and venous insufficiency with placebo (each 0.5\%), and syncope and irritability (each I.0\%) and feeling jittery, headache, tachycardia, cholecystitis, increased gamma-glutamyltransferase, fungal pneumonia, anxiety, and dyspnea (each 0.5\%) with LDX; study 2: anxiety, fibula fracture, blood pressure increase, and bradycardia (each 0.5\%) with placebo and initial insomnia, insomnia, optic atrophy, upper abdominal pain, lumbar vertebral fracture, increased heart rate, and rash (each 0.6\%) with LDX.

pathology compared with placebo, but only modest effects on weight (Reas and Grilo, 2014). Placebo-controlled studies of topiramate (Claudino et al, 2007; McElroy et al, 2003, 2007b) and sibutramine (Appolinario et al, 2003; Wilfley et al, 2008) have yielded significant effects on frequency of binge eating, other BED-related psychopathology, and weight. However, sibutramine is no longer marketed and the use of topiramate has been limited due to side effects, mainly cognitive impairment (Arif et al, 2009). The results of several small randomized, controlled trials of orlistat in BED have been mixed (Golay et al, 2005; Grilo et al, 2005; Grilo and White, 2013). Clinical trials to assess other agents for BED have been conducted, but there has only been at most a single placebo-controlled trial for any given medication and the sample sizes are small (Reas and Grilo, 2014). Statistically significant findings in favor of efficacy over placebo have been reported in a limited number of studies (Corwin et al, 2012; McElroy et al, 2006, 2007a; Pataky et al, 2013).

The safety and tolerability profile of LDX observed regarding overall TEAEs, TEAEs leading to discontinuation, vital signs, and ECGs were consistent with published reports in adults with BED (McElroy et al, 2015) or attention-deficit/ hyperactivity disorder (Adler et al, 2008; Wigal et al, 2010). Headache, insomnia, and dry mouth were the most frequently reported TEAEs with LDX in each study. Discontinuations due to TEAEs with LDX in these studies (6.3\% and $3.9 \%)$ are comparable to a published phase 2 study of LDX in BED (McElroy et al, 2015), to other clinical 
studies of LDX for which LDX is indicated (Adler et al, 2008; Wigal et al, 2010), and to other BED pharmacotherapy trials with other agents (Claudino et al, 2007; McElroy et al, 2003, 2007a, b).

These findings should be considered in light of potential limitations. Study participants were mainly women, white, overweight or obese, and did not have any current psychiatric comorbidities. As a result, caution is needed when generalizing to a more heterogeneous population. In addition, the short-term nature of the studies precludes extrapolations to the long-term efficacy, tolerability, and safety of LDX in individuals with BED. Also, comparisons of the efficacy of LDX in participants receiving $v s$ not receiving psychotherapy for BED were not conducted because the number of participants receiving psychotherapy for BED in the past or currently was small in both studies.

Taken together, the findings from both of these studies demonstrate that LDX may be an effective pharmacotherapy for BED. Further long-term studies are warranted to extend the results of these studies.

\section{FUNDING AND DISCLOSURE}

Susan L. McElroy, MD, had full access to all of the data in the study and takes responsibility for the integrity of the data and the accuracy of the data analysis. All authors made substantial contributions to the conception or design of the manuscript or the acquisition, analysis, or interpretation of data for the work; wrote the manuscript or revised it critically for important intellectual content; gave final approval of the version to be published; and are accountable for all aspects of the manuscript in ensuring that questions related to any part of the manuscript are appropriately investigated and resolved. Susan L. McElroy, MD, is a consultant to and has received grant support from Shire. In addition, she is also a consultant to or member of the scientific advisory boards of Alkermes, Bracket, Corcept, F. Hoffmann-LaRoche Ltd, MedAvante, Myriad, Naurex, Novo Nordisk, Sunovion, and Teva. She has also received grant support from the Agency for Healthcare Research \& Quality (AHRQ), Alkermes, AstraZeneca, Cephalon (now Teva), Forest, Lilly, Marriott Foundation, National Institute of Mental Health, Orexigen, Pfizer, Takeda, and Transcept. She is also an inventor on United States Patent No. 6,323,236 B2, Use of Sulfamate Derivatives for Treating Impulse Control Disorders and, along with the patent's assignee, University of Cincinnati (Cincinnati, OH), has received payments from Johnson \& Johnson, which has exclusive rights under the patent. James I. Hudson, MD, $\mathrm{ScD}$, has received consulting fees and grant support from Shire. In addition, he has received consulting fees from Genentech, Pronutria, Roche, and Sunovion; received grant support from Genentech; and received compensation for expert legal testimony from various law firms. M. Celeste FerreiraCornwell, PhD, Jana Radewonuk, MSc, Timothy Whitaker, $\mathrm{MD}$, and Maria Gasior, $\mathrm{MD}, \mathrm{PhD}$ are employees of Shire Development LLC and hold stock/stock options in Shire. Clinical research was funded by the sponsor, Shire Development LLC (Wayne, PA). Shire Development LLC also provided funding to Complete Healthcare Communications, LLC (Chadds Ford, PA), for support in writing and editing this manuscript. Although the sponsor was involved in the design, collection, management, analysis, interpretation, and fact checking of the data, the decision to submit it for publication in Neuropsychopharmacology was made by the authors.

\section{ACKNOWLEDGMENTS}

Under the direction of the authors, Stefan Kolata, $\mathrm{PhD}$, and Craig Slawecki, PhD, employees of Complete Healthcare Communications, LLC (CHC), provided writing and formatting assistance for this manuscript. Editorial assistance in the form of proofreading, copyediting, and fact checking was also provided by $\mathrm{CHC}$.

\section{REFERENCES}

Adler LA, Goodman DW, Kollins SH, Weisler RH, Krishnan S, Zhang Y et al (2008). Double-blind, placebo-controlled study of the efficacy and safety of lisdexamfetamine dimesylate in adults with attention-deficit/hyperactivity disorder. J Clin Psychiatry 69: 1364-1373.

American Psychiatric Association (2000). Diagnostic and Statistical Manual of Mental Disorders, 4th edn, Text Revision. American Psychiatric Association: Washington, DC.

American Psychiatric Association (2013). Diagnostic and Statistical Manual of Mental Disorders, 5th edn. American Psychiatric Association: Washington, DC.

Appolinario JC, Bacaltchuk J, Sichieri R, Claudino AM, Godoy-Matos A, Morgan C et al (2003). A randomized, doubleblind, placebo-controlled study of sibutramine in the treatment of binge-eating disorder. Arch Gen Psychiatry 60: 1109-1116.

Arif H, Buchsbaum R, Weintraub D, Pierro J, Resor SR Jr, Hirsch LJ (2009). Patient-reported cognitive side effects of antiepileptic drugs: predictors and comparison of all commonly used antiepileptic drugs. Epilepsy Behav 14: 202-209.

Claudino AM, de Oliveira IR, Appolinario JC, Cordas TA, Duchesne M, Sichieri R et al (2007). Double-blind, randomized, placebo-controlled trial of topiramate plus cognitive-behavior therapy in binge-eating disorder. J Clin Psychiatry 68: 1324-1332.

Corwin RL, Boan J, Peters KF, Ulbrecht JS (2012). Baclofen reduces binge eating in a double-blind, placebo-controlled, crossover study. Behav Pharmacol 23: 616-625.

Fairburn CG, Beglin SJ (1994). Assessment of eating disorders: interview or self-report questionnaire? Int $J$ Eat Disord 16: 363-370.

First M, Williams J, Spitzer R, Gibbon M (2007). Structured Clinical Interview for DSM-IV-TR Axis I Disorders, Clinical Trials Version (SCID-CT). Biometrics Research, New York State Psychiatric Institute: New York, NY, USA.

Golay A, Laurent-Jaccard A, Habicht F, Gachoud JP, Chabloz M, Kammer A et al (2005). Effect of orlistat in obese patients with binge eating disorder. Obes Res 13: 1701-1708.

Goodman WK, Price LH, Rasmussen SA, Mazure C, Fleischmann RL, Hill CL et al (1989). The Yale-Brown Obsessive Compulsive Scale. I. Development, use, and reliability. Arch Gen Psychiatry 46: 1006-1011.

Grilo CM, Masheb RM, Salant SL (2005). Cognitive behavioral therapy guided self-help and orlistat for the treatment of binge eating disorder: a randomized, double-blind, placebocontrolled trial. Biol Psychiatry 57: 1193-1201.

Grilo CM, White MA (2013). Orlistat with behavioral weight loss for obesity with versus without binge eating disorder: randomized placebo-controlled trial at a community mental health center serving educationally and economically disadvantaged Latino/as. Behav Res Ther 51: 167-175. 
Guy W (1976). Clinical Global Impressions. ECDEU Assessment Manual for Psychopharmacology, US Department of Health, Education, and Welfare; Public Health Service Alcohol, Drug Abuse and Mental Health Administration; NIMH Psychopharmacology Research Branch: Rockville, MD, pp 218-222.

Hudson JI, Hiripi E, Pope HG Jr, Kessler RC (2007). The prevalence and correlates of eating disorders in the National Comorbidity Survey Replication. Biol Psychiatry 61: 348-358.

Hudson JI, Lalonde JK, Coit CE, Tsuang MT, McElroy SL, Crow SJ et al (2010). Longitudinal study of the diagnosis of components of the metabolic syndrome in individuals with binge-eating disorder. Am J Clin Nutr 91: 1568-1573.

Kessler RC, Berglund PA, Chiu WT, Deitz AC, Hudson JI, Shahly V et al (2013). The prevalence and correlates of binge eating disorder in the World Health Organization World Mental Health Surveys. Biol Psychiatry 73: 904-914.

Little RJ, D'Agostino R, Cohen ML, Dickersin K, Emerson SS, Farrar JT et al (2012). The prevention and treatment of missing data in clinical trials. $N$ Engl J Med 367: 1355-1360.

Masheb RM, Grilo CM (2004). Quality of life in patients with binge eating disorder. Eat Weight Disord 9: 194-199.

McElroy SL, Arnold LM, Shapira NA, Keck PE Jr, Rosenthal NR, Karim MR et al (2003). Topiramate in the treatment of binge eating disorder associated with obesity: a randomized, placebocontrolled trial. Am J Psychiatry 160: 255-261.

McElroy SL, Guerdjikova A, Kotwal R, Welge JA, Nelson EB, Lake KA et al (2007a). Atomoxetine in the treatment of bingeeating disorder: a randomized placebo-controlled trial. J Clin Psychiatry 68: 390-398.

McElroy SL, Hudson JI, Capece JA, Beyers K, Fisher AC, Rosenthal NR et al (2007b). Topiramate for the treatment of binge eating disorder associated with obesity: a placebocontrolled study. Biol Psychiatry 61: 1039-1048.

McElroy SL, Hudson JI, Mitchell JE, Wilfley D, Ferreira-Cornwell MC, Gao J et al (2015). Efficacy and safety of lisdexamfetamine for treatment of adults with moderate to severe binge-eating disorder: a randomized clinical trial. JAMA Psychiatry 72: 235-246.

McElroy SL, Kotwal R, Guerdjikova AI, Welge JA, Nelson EB, Lake KA et al (2006). Zonisamide in the treatment of binge eating disorder with obesity: a randomized controlled trial. J Clin Psychiatry 67: 1897-1906.

McGregor C, Srisurapanont M, Mitchell A, Longo MC, Cahill S, White JM (2008). Psychometric evaluation of the Amphetamine Cessation Symptom Assessment. J Subst Abuse Treat 34: 443-449.

Pataky Z, Gasteyger C, Ziegler O, Rissanen A, Hanotin C, Golay A (2013). Efficacy of rimonabant in obese patients with binge eating disorder. Exp Clin Endocrinol Diabetes 121: 20-26.

Pennick M (2010). Absorption of lisdexamfetamine dimesylate and its enzymatic conversion to d-amphetamine. Neuropsychiatr Dis Treat 6: 317-327.

Posner K, Brown GK, Stanley B, Brent DA, Yershova KV, Oquendo MA et al (2011). The Columbia-Suicide Severity Rating Scale: initial validity and internal consistency findings from three multisite studies with adolescents and adults. Am J Psychiatry 168: $1266-1277$.

Reas DL, Grilo CM (2014). Current and emerging drug treatments for binge eating disorder. Expert Opin Emerg Drugs 19: 99-142.

Siddiqui O (2011). MMRM versus MI in dealing with missing dataa comparison based on 25 NDA data sets. J Biopharm Stat 21: 423-436.

Striegel-Moore RH, Dohm FA, Wilfley DE, Pike KM, Bray NL, Kraemer HC et al (2004). Toward an understanding of health services use in women with binge eating disorder. Obes Res 12: 799-806.

Vocks S, Tuschen-Caffier B, Pietrowsky R, Rustenbach SJ, Kersting A, Herpertz S (2010). Meta-analysis of the effectiveness of psychological and pharmacological treatments for binge eating disorder. Int J Eat Disord 43: 205-217.

Vyvanse (lisdexamfetamine dimesylate) (2015). Full Prescribing Information. Shire US Inc: Wayne, PA.

Wigal T, Brams M, Gasior M, Gao J, Squires L, Giblin J et al (2010). Randomized, double-blind, placebo-controlled, crossover study of the efficacy and safety of lisdexamfetamine dimesylate in adults with attention-deficit/hyperactivity disorder: novel findings using a simulated adult workplace environment design. Behav Brain Funct 6: 34.

Wilfley DE, Crow SJ, Hudson JI, Mitchell JE, Berkowitz RI, Blakesley V et al (2008). Efficacy of sibutramine for the treatment of binge eating disorder: a randomized multicenter placebocontrolled double-blind study. Am J Psychiatry 165: 51-58.

(c) (i) $(9)$ This work is licensed under a Creative Commons Attribution-NonCommercial-NoDerivs $\quad 4.0$ International License. The images or other third party material in this article are included in the article's Creative Commons license, unless indicated otherwise in the credit line; if the material is not included under the Creative Commons license, users will need to obtain permission from the license holder to reproduce the material. To view a copy of this license, visit http://creativecommons.org/licenses/by-nc-nd/4.0/

Supplementary Information accompanies the paper on the Neuropsychopharmacology website (http://www.nature.com/npp) 